\title{
La pluralización del verbo haber existencial en Madrid: ¿etapas iniciales de un cambio lingüístico?
}

\author{
Florentino Paredes García ${ }^{1}$ \\ Universidad de Alcalá
}

\begin{abstract}
Resumen
La alternancia singular/plural de la concordancia entre el verbo haber con valor existencial y el argumento con que se combina es uno de los fenómenos que presenta variación en el español hablado a ambos lados del Atlántico. Este artículo presenta la investigación realizada en la comunidad de habla de Madrid, una ciudad tradicionalmente exenta de este fenómeno innovador, a partir del análisis de las 108 entrevistas semidirigidas que forman los dos corpus PRESEEA de la ciudad, correspondientes al distrito de Salamanca y a los de Vallecas y Puente de Vallecas. Para el análisis de la variable lingüística dependiente (singular/plural) se ha seguido la propuesta de codificación de la variable elaborada en el marco del PRESEEA, que considera la posible incidencia de 23 variables independientes: once lingüísticas, cuatro estilísticas y ocho sociológicas. Los resultados obtenidos indican que la pluralización, aunque es un fenómeno aún poco frecuente, está introduciéndose lentamente en la variedad madrileña. Por ello, además de analizar la incidencia de los factores
\end{abstract}

1Para correspondencia, dirigirse a: Florentino Paredes García (florentino.paredes@uah. es), Departamento de Filología, Comunicación y Documentación, Universidad de Alcalá, c/, Trinidad, 5, 28801- Alcalá de Henares - Madrid, España. 
lingüísticos, estilísticos y sociales, se ha estudiado con detalle la fase en la que se encuentra la innovación lingüística, la posible incidencia de ciertas restricciones cognitivas y las características de los hablantes que han introducido la construcción pluralizada.

Palabras clave: variación sintáctica, verbo haber existencial, cambio lingüístico, español hablado, comunidad de habla de Madrid.

The Pluralization of the eXistential Verb haber in MADRID. INITIAL STAGES OF A LINGUISTIC CHANGE?

\begin{abstract}
The singular/plural alternation of the concordance between the verb haber with existential meaning and its argument is a phenomenon that changes according to the variety of Spanish used -that will differ depending on the side of the Atlantic is used. This article presents the research carried out in Madrid, a linguistic community traditionally exempt from this innovative phenomenon, based on the analysis of 108 semi-directed interviews that conform the PRESEEA corpora and that correspond to the Salamanca and Vallecas-Puente de Vallecas districts. The analysis of the dependent linguistic variable (singular/ plural) has been carried out according to the guidelines proposed within the framework of PRESEEA. This framework considers the potential impact of 23 independent variables: eleven linguistic, four stylistic, and eight sociological. The results indicate that the pluralization, although still rare, is slowly being introduced in the variety of Spanish spoken in Madrid. Therefore, in addition to the analysis of the incidence of the stylistic, social and linguistic factors, the linguistic innovation stage has also been studied in detail, as well as the potential impact of the cognitive constraints and characteristics of the speakers that have introduced these pluralized constructions.
\end{abstract}

Keywords: syntactic variation, existential haber, linguistic change, spoken Spanish, Madrid speech community.

Recibido: 01/06/2016 Aceptado: 06/06/2016 


\section{INTRODUCCIÓN ${ }^{2}$}

La concordancia de número entre el verbo haber con valor existencial y el argumento con el que se combina es uno de los fenómenos gramaticales variables del español actual que más atención ha recibido. Según la Nueva Gramática de la Lengua Española (NGLE), el verbo haber se usa como impersonal para referirse a fenómenos atmosféricos (hay niebla), cronológicos (hubo épocas mejores) y a la existencia de cualquier otra realidad (había dos personas esperando) (RAE y ASALE 2009: 3063). No obstante, en muchas variedades del español se ha producido un cambio en el régimen sintáctico del verbo, que se pone de manifiesto en la concordancia entre el verbo y la FN con la que se combina (habian muchas personas, hubieron incendios).

En los estudios sobre este asunto se han seguido básicamente dos enfoques, el prescriptivo y el funcionalista (Bentivoglio y Sedano 1989:60), y el debate se ha polarizado sobre todo en el estatus funcional del argumento verbal. La visión tradicional considera impersonal el verbo cuando tiene valor existencial o presentacional, por lo que carece de sujeto y la FN desempeña la función sintáctica de complemento directo, como muestra la sustitución pronominal con clíticos de objeto (Acudieron muchas personas y las habia de todas las nacionalidades, Ese año se produjeron incendios en la ciudad y los hubo también en el campo). Sobre esta consideración se establece la norma que reprueba la concordancia, al considerarla un uso desviado respecto a la construcción primigenia. El segundo enfoque, en cambio, se interesa por describir el fenómeno e identificar las causas que explican el cambio y los factores que lo favorecen o lo restringen. Los hablantes que concuerdan la FN que acompaña al verbo la interpretan como sujeto de la oración (Martínez 1999: 2770-71), no como objeto directo. La elección de una u otra construcción no supone diferencias sustanciales de interpretación, pues en cualquiera de los dos casos con la FN nominal el hablante proporciona información nueva respecto a las creencias o conocimientos del oyente (Claes 2014a). Se ha constatado también que la pluralización de haber es un fenómeno innovador, que en el español de América surgió hacia los siglos XVII y XVIII (Fontanella de Weinberg 1992) y que en la actualidad avanza

\footnotetext{
Este trabajo se inscribe en las investigaciones del proyecto "Estudio complementario de los patrones sociolingüísticos y los procesos de integración sociolingüística en el español de Madrid (ECOPASIS-Madrid)" (ref. FFI2015-68171-C5-4-P), financiado por el Ministerio de Economía y Competitividad de España.
} 
a diferentes ritmos en las variedades del español. Un aspecto que hace más interesante su estudio son las repercusiones sociolingüísticas, ya que la pluralización se ha asociado a características sociológicas de los hablantes y a estilos de habla, señalándose al respecto que "el uso cuantitativamente diferenciado de las variantes le permite al hablante ubicarse socialmente" (Claes 2014a: 342).

Con objeto de contribuir a una perspectiva comparada que permita establecer con exactitud los grados de avance y difusión del fenómeno en el ámbito hispánico, los equipos del Proyecto para el estudio sociolingüístico del español de España y América (PRESEEA) acordaron priorizar la variación del verbo haber con valor existencial. Este trabajo, por tanto, constituye la aportación del equipo de Madrid-Alcalá a esa tarea conjunta. El objetivo es analizar en la comunidad de habla de Madrid la concordancia del verbo haber con valor existencial y argumento plural, para lo cual se seguirá en lo esencial la citada propuesta de codificación. Si se constata que la pluralización está iniciando su desarrollo y difusión en esta comunidad de habla, las hipótesis de las que se parte son dos: a) que sobre la difusión de la variable actuarían factores lingüísticos, estilísticos, cognitivos y sociales que frenarían o difundirían la innovación y b) que Madrid se encontraría en un proceso de convergencia con el resto de variedades del español innovadoras en este punto. Por ello, el interés por conocer lo que sucede en la capital de España respecto a este fenómeno estriba, más allá del interés intrínseco del conocimiento de la situación lingüística de la ciudad, en que permitirá observar cómo se introduce la variación en una comunidad de habla que tradicionalmente ha estado exenta de formas concordadas, al menos en la lengua de los hablantes cultos (De Mello 1991). El análisis de la lengua hablada de los madrileños permitirá, por una parte, ver en qué estadio se encuentra el fenómeno o qué fases se han cumplido y situar Madrid en relación con otras variedades del español y, por otra, determinar si en el proceso de difusión de este cambio gramatical intervienen algunas restricciones cognitivas generales, tal como han señalado algunos autores para otras comunidades de habla hispanas (Claes 2014a). Por último, y en esta misma línea, el trabajo permitirá analizar con detalle las características individuales de los hablantes que promueven el cambio en la comunidad de habla madrileña para determinar quiénes son, en qué medida se adhieren al cambio, qué vinculación tienen con zonas hispanohablantes en las que el proceso de pluralización está más avanzado, o en qué medida afecta su participación en el mercado lingüístico en el uso concordado. 


\section{MARCO TEÓRICO Y ESTADO DE LA CUESTIÓN}

Desde la perspectiva descriptivo-explicativa, el debate sobre el estatus del argumento que aparece con el verbo ha sido uno de los asuntos centrales, como se ha señalado. En la evolución del verbo latino HABĒRE han actuado causas sintácticas y semánticas (Luque Moreno 1978, González Calvo 2002, Hernández Díaz 2006). El verbo se utilizaba para indicar posesión y era personal y transitivo en latín, uso que pasó al español y sobrevivió al menos hasta el siglo XVII (Fontanella de Weinberg 1992:32). Pero junto a este uso, en latín tardío surgió una construcción nueva en la que HABĒRE se usaba para indicar existencia o presencia, en cuyo caso aparecía en tercera persona de singular seguido de acusativo. En el paso al español esta construcción dio lugar a los usos impersonales actuales que, en una evolución posterior, derivan en construcciones en las que haber concuerda en número y persona con su argumento ${ }^{3}$.

Una de las aportaciones teóricas más recientes para explicar este cambio ha surgido de la lingüística cognitiva. Desde esta perspectiva, Claes (2014a, 2014b) parte de la idea de que en español la representación mental de haber existencial (o presentacional, según su terminología) se vincula con dos construcciones, una en la que el argumento nominal de haber es objeto y otra en la que el argumento es sujeto. La elección de una u otra construcción está determinada por tres restricciones cognitivas generales: la marcadez de la codificación, la disuasión estadística y el priming estructural.

La marcadez de la codificación-idea basada en Langacker (1991)-, supone que la elección por parte del hablante de una determinada función sintáctica está condicionada por la semejanza con el prototipo de dicha función. En el caso de la pluralización de haber, la conceptualización del argumento como sujeto estará asociada a los rasgos que caracterizan prototípicamente a los sujetos, en especial a la agentividad. Tradicionalmente, la agentividad se ha asociado a los SSNN animados o humanos, pero Claes (2014b: 5-6) precisa que está relacionada con la capacidad de la entidad representada en la FN para convertirse en "cabeza de una cadena de acciones", esto es, para transferir energía y desencadenar un evento que origine cambios de estado interno a otra entidad, y no como "cola de la cadena de acciones", es

\footnotetext{
3 Las propiedades sintácticas de haber se describen en Fernández Soriano y Táboas Baylín 1999: 1754-1759. Para un análisis más detallado de la evolución histórica de la sintaxis y los valores semánticos del verbo pueden verse González Calvo 2002 o Hernández Díaz 2006.
} 
decir, como elemento al que se transfiere la energía y en el que tiene lugar el cambio interno. De este modo, entre dos entidades como conductor y víctima, ambas caracterizadas como animadas y humanas, la primera se concibe más fácilmente como agente, esto es, como "cabeza" capaz de desencadenar acciones, mientras que la segunda se tiende a concebir como no agente, es decir, como "cola" o resultado de la energía transferida. Por ello, una entidad como conductor se conceptualiza como agente potencial, y por tanto como sujeto prototípico, más fácilmente que víctima.

La disuasión estadística (Goldberg 2006: 94) es una restricción cognitiva que tiene que ver con la frecuencia de las estructuras lingüísticas. Según esta hipótesis, la exposición a las estructuras lingüísticas a que se somete el hablante determina sus representaciones mentales. Las formas que ocurren en más de una construcción originan representaciones independientes de diversos esquemas abstractos, mientras que aquellas que ocurren en una sola construcción se almacenan como casos particulares de esquemas abstractos. El diferente modo de almacenamiento tiene consecuencias en el acceso a estas construcciones y en su recuperación. Aplicado al caso de haber, la restricción afecta al tiempo en que se actualiza el verbo en su valor presentacional. Los estudios diacrónicos cuantitativos muestran que las formas hay y hubo eran las más frecuentes para el valor existencial, mientras que había no solo era menos habitual para este valor sino que también aparece en otros tipos de construcciones. En consecuencia, en el inicio del cambio en los siglos XVII o XVIII, es lógico que el hablante evitara la pluralización para referirse a situaciones en presente o en pretérito indefinido, dado que disponía de un esquema prefabricado para estos casos, mientras que en el caso de habia no actuaba esta restricción, por lo que era más posible la concordancia en plural.

El priming estructural, por último, es un concepto surgido desde la psicolingüística según el cual el hablante recicla inconscientemente las construcciones abstractas que ha utilizado u observado y las pone de manifiesto en las producciones lingüísticas. Las investigaciones sugieren que la permanencia del efecto del priming se mantiene activo al menos con una distancia de diez verbos conjugados e independientemente de si el hablante produce la oración o solo la procesa.

Hay que señalar que estas tres restricciones descritas actúan en orientación contraria respecto a la difusión del fenómeno: la restricción de marcadez de la codificación y el priming estructural tienden a extender la construcción pluralizada a nuevos contextos y a nuevas representaciones mentales, mientras que la restricción de la disuasión estadística refrena la expansión de la concordancia en plural. 
Por otra parte, respecto al proceso de generalización del fenómeno, Hernández Díaz (2006: 1151) sugiere que en el proceso de cambio pueden diferenciarse cuatro etapas, cada una de las cuales supone un progresivo alejamiento de las formas más frecuentes o prototípicas del verbo:

1) Innovación agazapada: en las primeras etapas del cambio, la concordancia recae sobre un verbo auxiliar: tienen que haber.

2) Innovación de menor alteración: en una segunda etapa, los casos de concordancia afectan directamente al verbo haber, pero los cambios que conlleva son mínimos, como la agregación de -n: habían, habrán, habrian.

3) Innovación de mayor alteración: en una tercera etapa, la concordancia se extiende a tiempos verbales en los que los cambios formales entre singular y plural son mayores: hubieron. También se integran en esta etapa los cambios en los que las formas de haber incluyen al hablante: habemos, habíamos, habremos.

4) Innovación extrema: en el grado extremo del cambio, la concordancia se extiende al presente generando formas extrañas a la conjugación española, como haen (Montes Giraldo 1982) o hayn (Kany 1971: 257).

No obstante, el proceso descrito está basado casi exclusivamente en la difusión de las construcciones innovadoras en la lengua escrita, por lo que habrá que ver si en la extensión de las construcciones pluralizadas en la lengua oral y en su generalización en el seno de una comunidad de habla se cumplen estas fases o si, por el contrario, para estos casos hay que buscar nuevas hipótesis explicativas.

En cuanto a la difusión geográfica, parece haber unanimidad en considerar que la concordancia de haber en plural está hoy en expansión en español (RAE y ASALE 2009: 3063). En numerosas variedades del español esta concordancia se ha presentado como un cambio en curso, que se desarrolla especialmente en territorio americano aunque avanza a diferentes ritmos y presenta diferente intensidad (De Mello 1991; Bentivoglio y Sedano 1989, Lastra y Martín Butragueño 2015). Dentro de España se ha venido sosteniendo tradicionalmente que la pluralización de haber es característica de las zonas de contacto con el catalán (Seco 1986, RAE y ASALE 2009, Gómez Molina 2013), señalándose incluso que la variante pluralizada se realiza de forma cuasi categórica (Blas Arroyo 2005: 558). Para otros, en cambio, la concordancia en plural es un fenómeno habitual también en otras regiones españolas, entre ellas la zona canaria (Catalán 1964, Almeida y Díaz Ayalón 1989, Samper y Hernández 2012), Aragón, Murcia y las áreas orientales de Castilla-La Mancha y Andalucía (Llorente 1980: 31). Las noticias sobre la 
pluralización de haber existencial apuntan a que el fenómeno también se encuentra en el centro peninsular (Lorenzo 1971: 223), si bien se carece de más precisiones que permitan delimitar el alcance geográfico y social en este territorio.

En lo que atañe en particular a la ciudad de Madrid, los estudios sobre la pluralización de haber existencial en la ciudad no han sido muchos. En el trabajo de De Mello (1991), basado en la lengua hablada culta del español de once ciudades hispanohablantes, nueve americanas y dos españolas, se ofrece un análisis cuantitativo de los usos de haber existencial que muestra que los usos pluralizados se dan en todas las ciudades americanas, especialmente en Lima, La Paz, Caracas, Santiago y San Juan, pero no encuentra ningún caso en las encuestas de Madrid ni en las de Sevilla.

Años antes, sin embargo, sirviéndose de esas mismas encuestas del proyecto para el estudio coordinado de la norma culta española, Quilis había encontrado en Madrid algún testimonio del fenómeno, aunque su producción fuera escasa:

En nuestros materiales, de 2.384 construcciones registradas de este tipo, sólo tenemos dos que presenten construcción irregular, con haber: "Habían muchas injusticias sociales", "Habían programas..." (Quilis 1983: 94).

De esas dos muestras recogidas, una pertenece a un joven de entre quince y veinte años y la otra a una mujer de más de 55 años. Así pues, entre los hablantes cultos madrileños de finales de los 60 y principios de los 70 (las encuestas se iniciaron entre septiembre y diciembre de 1968), la variante pluralizada de haber apenas alcanzaba el 0,08\%, aunque hay que tener en cuenta que en este porcentaje se incluyen también los casos de hay.

Por último, desde el punto de vista de la estratificación social, la concordancia en plural tradicionalmente se ha considerado rasgo propio de hablantes incultos o poco instruidos (Lope Blanch 1953, Flórez 1946), aunque no han faltado estudios que han considerado que la pluralización de haber se encuentra también entre hablantes cultos (Kany 1951, De Mello 1991). En la $N G L E$ se relaciona el empleo de las formas pluralizadas con el lenguaje de los medios de comunicación, donde "la proporción de los usos concordados es mayor en la lengua periodística que en otro tipo de textos" (2009: 3063, vid. también Regueiro Rodríguez 2008), y Samper y Hernández (2012: 745) encuentran casos de pluralización incluso en el Boletín Oficial de la comunidad autónoma canaria. En el trabajo de Lastra y Martín Butragueño (2015) se resumen los resultados de los principales estudios sobre la pluralización de haber realizados con datos de lengua hablada. Aunque los resultados de los estudios no son coincidentes, entre 
otras razones por las diferencias metodológicas, los autores resaltan que en la mayor parte de los trabajos aparecen muchos factores sociales como relevantes: en diversas ciudades la pluralización se ha asociado con el nivel socioeducativo bajo, con los jóvenes y adultos y con las mujeres, aunque en otras ciudades ha sido el grupo masculino el más propiciador del fenómeno. En cuanto a los factores lingüísticos, en muchos casos se señala que la pluralización sucede especialmente cuando la FN nominal posee el rasgo + humano, cuando el argumento está definido y cuando pragmáticamente es el tópico oracional.

\section{METODOLOGÍA}

En este trabajo se han tomado en cuenta las 108 encuestas completas que conforman los corpus PRESEEA en la ciudad de Madrid, uno sobre el distrito de Salamanca y otro sobre los distritos de Vallecas y Puente de Vallecas (cf. Cestero, Molina y Paredes 2015: 33-46).

Siguiendo los criterios del PRESEEA (Moreno Fernández 1996), se recurrió a un muestreo por cuotas de afijación uniforme, atendiendo como criterios de preestratificación al sexo, la edad y el nivel de instrucción, con tres representantes por casilla.

\begin{tabular}{|c|c|c|c|c|c|c|}
\cline { 2 - 7 } \multicolumn{1}{c|}{} & \multicolumn{2}{|c|}{ Generación 1 } & \multicolumn{2}{c|}{ Generación 2 } & \multicolumn{2}{c|}{ Generación 3 } \\
\cline { 2 - 7 } & $\mathrm{H}$ & $\mathrm{M}$ & $\mathrm{H}$ & $\mathrm{M}$ & $\mathrm{H}$ & $\mathrm{M}$ \\
\hline Nivel de instrucción 1 & 3 & 3 & 3 & 3 & 3 & 3 \\
\hline Nivel de instrucción 2 & 3 & 3 & 3 & 3 & 3 & 3 \\
\hline Nivel de instrucción 3 & 3 & 3 & 3 & 3 & 3 & 3 \\
\hline
\end{tabular}

En cada uno de los distritos se han realizado 54 entrevistas, de aproximadamente una hora de duración, Posteriormente los sujetos fueron agrupados atendiendo a diversos criterios de postestratificación, como la clase social, el modo de vida. Para este trabajo podía ser de interés también ver si los datos de origen del informante, de su cónyuge o de sus 
ascendientes podían tener algún tipo de influencia en la aparición de las formas concordadas de haber.

En el análisis de la variable se ha seguido la propuesta de codificación elaborada por José Ramón Gómez Molina con la colaboración de Paola Bentivoglio y Clara E. Hernández Cabrera (Gómez Molina 2013:118129, disponible también en http://preseea.linguas.net/), añadiendo algunas variables relativas a las características sociológicas de los madrileños para observar su posible relación con las realizaciones concordadas de haber en plural.

La variable dependiente analizada ha sido la realización singular o plural del verbo, tomando en cuenta los casos de haber acompañado de una FN plural o de una FN singular colectiva. Siguiendo el modelo de codificación señalado, se ha analizado la posible incidencia de 23 variables independientes, once lingüísticas, cuatro estilísticas y ocho sociológicas. Son las siguientes:

- Variables lingüísticas: (1) estructura verbal, (2) valor semántico de la FN, (3) animacidad de la FN, (4) definitud de la FN, (5) forma léxica de la FN, (6) posición de la FN respecto de haber, (7) marcas de pluralidad de la FN, (8) tiempo y modo verbal, (9) intercalación de elementos entre el V y el argumento, (10) tipo de oración y (11) polaridad.

- Variables estilísticas: (12) especialización del discurso (campo o tema de conversación), (13) tipo de discurso, (14) formalidad (tenor y grado de proximidad entre informante y entrevistador) y (15) formalidad (tenor y relación por edad entre informante y entrevistador).

- Variables sociológicas: (16) sexo, (17) edad, (18) nivel de estudios, (19) modo de vida, (20) clase social (variable postestratificada obtenida a partir del nivel de estudios y la profesión, Cestero, Molina y Paredes, 2015:35-36), (21) distrito de residencia, (22) localidad de origen y (23) ascendencia (existencia o no de cónyuge, padres o abuelos originarios de región concordante).

El análisis de los datos ha atendido a las dimensiones cualitativa y cuantitativa. La escasez de ejemplos de discordancia ha permitido un análisis pormenorizado de cada uno de los casos encontrados, con objeto de encontrar pautas que permitan explicar el modo en que se introduce y difunde la innovación en el seno de la comunidad madrileña. A pesar de la escasez de ejemplos, se ha podido realizar también un acercamiento cuantitativo $\mathrm{y}$, siguiendo la práctica habitual de la metodología sociolingüística, se han 
empleado tanto procedimientos de estadística simple (frecuencia absoluta y relativa, deviación típica, rango), como pruebas de estadística inferencial, que permiten hacer proyecciones acerca de la representatividad de los datos. Para ello se han empleado los programas SPSS y GOLDVARB 2.0, habituales en los análisis estadísticos de sociolingüística.

\section{ANÁLISIS DE LOS RESULTADOS}

En las encuestas del corpus PRESEEA de Madrid hemos encontrado 3310 casos en los que el verbo haber se usa para expresar valor existencial. De ellos, la mayoría ( $\mathrm{N}=2740 ; 82,8 \%)$ corresponde a la tercera persona del presente de indicativo, hay. Como cabía esperar y como sucede también en todos los estudios sobre el español de España (cf. Gómez Molina 2013: 120, Samper y Hernández 2012:748; Blas Arroyo 2016: 7) y en algunos de América (Lastra y Martín Butragueño 2015: 3), no se han documentado formas pluralizadas del tipo hayn, hain o haen, que se sitúan en el grado más extremo del proceso de cambio y suponen una marcación de plural percibida como ajena al sistema gramatical o muy alejada de él. Por esta razón, en este trabajo han quedado excluidos de los análisis, como se ha hecho también en los estudios citados.

Tabla 1. Casos de haber existencial con FN plural en Madrid

\begin{tabular}{|l|r|r|}
\cline { 2 - 3 } \multicolumn{1}{c|}{} & $\mathrm{N}$ & $\%$ \\
\hline Hay & 2740 & 82,8 \\
\hline Resto de formas del verbo & 568 & 17,2 \\
\hline Total & 3310 & 100 \\
\hline
\end{tabular}

La variación en la comunidad de habla madrileña queda restringida, por tanto, a los 568 casos en los que el verbo haber aparece en pretérito imperfecto, condicional, pretérito perfecto o formando parte de una perífrasis. En este subconjunto, la pluralización se ha encontrado en doce casos, lo que supone una presencia porcentual de tan solo el $2,1 \%$. Se han tenido en cuenta todos los casos en los que hay pluralización, incluidos aquellos en los que el hablante se corrige posteriormente, como vemos en el ejemplo (8). Como es evidente, la autocorrección solo indica que el hablante es consciente de que se trata de una forma no preferida, pero sirve de indicador de que el 
proceso de pluralización se ha iniciado en la comunidad de habla, aunque sea de manera incipiente ${ }^{4}$.

Tabla 2. Casos de variación de haber con FN plural

\begin{tabular}{|l|r|r|}
\cline { 2 - 3 } \multicolumn{1}{c|}{} & $\mathrm{N}$ & $\%$ \\
\hline No concordancia & 556 & 97,9 \\
\hline Concordancia & 12 & 2,1 \\
\hline Total & 568 & 100 \\
\hline
\end{tabular}

Dada la escasez de ejemplos de haber concordado en plural, las conclusiones que puedan extraerse acerca de los condicionamientos lingüísticos, estilísticos y sociales que afectan al proceso de cambio en la comunidad de habla madrileña han de ser tomadas con cautela. De hecho, las pruebas de estadística inferencial que se han aplicado para determinar la incidencia de las variables en la variable dependiente casi nunca han superado el umbral de significación estadística, establecido convencionalmente en 0,05 , lo que implica que no se puede descartar la hipótesis nula de independencia o, de otra forma, que los datos obtenidos pueden deberse al azar. No obstante, el interés del análisis detallado de estos casos reside en la posibilidad de encontrar algunas pistas o indicios acerca del modo en que se produce la difusión del cambio, justo en el momento en que parece estar empezando a tener lugar.

Si se comparan los datos obtenidos en este estudio con los de otros estudios de metodología similar que analizan la lengua hablada a finales del siglo Xx o principios del XXI, se puede observar que Madrid continúa situado en el extremo más conservador entre las ciudades hispanohablantes, a pesar de que se haya producido un avance respecto a lo que señalaban Quilis (1983) o De Mello (1991) para la década de los 70 del siglo pasado.

Tabla 3. Pluralización del verbo haber existencial en algunas ciudades hispanohablantes en torno al año 2000

\begin{tabular}{|l|r|c|}
\cline { 2 - 3 } \multicolumn{1}{c|}{} & $\mathrm{F} / \mathrm{N}$ & $\%$ \\
\hline Caracas (D’Aquino 2004) & $477 / 804$ & 63 \\
\hline Castellón (Blas Arroyo 2016) & $257 / 495$ & 52 \\
\hline
\end{tabular}

4 Blas Arroyo (2016: 2) encuentra en las encuestas del PRESEEA de Alcalá de Henares tan solo siete ejemplos de 361 ocurrencias de haber existencial, es decir, apenas el 1\% del total. 


\begin{tabular}{|l|c|c|}
\hline La Habana (Claes 2014b) & $934 / 2093$ & 44,6 \\
\hline $\begin{array}{l}\text { Santo Domingo (Claes } \\
\text { 2014b) }\end{array}$ & $859 / 1841$ & 46,7 \\
\hline $\begin{array}{l}\text { Valencia, España (Gómez } \\
\text { Molina 2013) }\end{array}$ & $127 / 275$ & 46 \\
\hline $\begin{array}{l}\text { San Juan, Puerto Rico (Claes } \\
\text { 2014b) }\end{array}$ & $684 / 1655$ & 41,3 \\
\hline $\begin{array}{l}\text { Las Palmas de Gran Canaria, } \\
\text { España (Sampery Hernández } \\
\text { 2012) [solo hablantes cultos] }\end{array}$ & $32 / 156$ & 20,5 \\
\hline $\begin{array}{l}\text { México, México (Lastra y } \\
\text { Martín Butragueño 2015) }\end{array}$ & $47 / 561$ & 8,4 \\
\hline Madrid, España (este estudio) & $12 / 568$ & 2,1 \\
\hline
\end{tabular}

\subsection{LAS VARIABLES LINGÜÍSTICAS}

Entre los doce casos de pluralización de haber, se encuentran dos ejemplos, ambos en pretérito imperfecto, en los que el verbo forma parte de una perífrasis (ejemplos 1-2):

1) a mí me avisó un amigo de aquí que iban a haber oposiciones cuando el año noventa y dos // que iba a haber oposiciones

[MADR-SAL_H12_19] $]^{5}$

2) luego también / ya nos vinimos en / en ese tiempo que empezaban a haber otros problemas

[MADR-SAL_M32_35]

También en dos ocasiones (ejemplos 3-4) la pluralización se produce con el verbo en pretérito perfecto compuesto:

3) una nieve que cuaje es muy difícil hoy día yo creo vamos son contadas las nevadas que han habido últimamente en Madrid

[MADR-SAL_H33_15]

5 La codificación identifica el distrito al que corresponde la entrevista (SAL $=$ Distrito de Salamanca, $\mathrm{VAL}=$ Distritos de Vallecas y Puente de Vallecas) el sexo del hablante $(\mathrm{H}=$ hombre, $\mathrm{M}=$ mujer $)$, la edad $(1=$ menor de 35 años, $2=$ entre 35 y 54 años y $3=$ mayor de 54 años) y el nivel de instrucción ( $1=$ estudio primarios, $2=$ estudios secundarios y $3=$ estudios universitarios). Las dos últimas cifras corresponden al número de encuesta. 
4) y que han habido épocas / pues / $\mathrm{mmm} /$ pues no sé de de las cuales no hay constancia digamos / archivada

[MADR-VAL_H23_32]

En el resto de ocasiones, la concordancia del verbo y su argumento sucede en pretérito imperfecto (ejemplos 4-11):

5) bueno pues eeh han cambiado las estructuras de las calles / antes habían bulevares ahora / pues han quedado en calles únicas de direcciones

6) habían como hoteles así

[MADR-SAL_H23_08]

[MADR-VAL_M12_12]

7) incluso en / verano / las tormentas / aquellas que habían / ya no las hay

[MADR-VAL_H22_26]

8) porque habían cuevas / en $<$ ininteligible/ $>$ había cuevas $/ /<$ ruido = "golpe" $/>$ hasta el pueblo Vallecas

[MADR-VAL_H31_38]

9) era el río Pelotas / donde todo el mundo lo tiraba allí // y habían / unos puentecitos de madera / donde tenías que pasar

[MADR-VAL_H31_38]

10) digo "así que llevaros bien / y de políticas aquí nada / aquí se viene a trabajar"// porque habían dos compañeros / que eran / inspectores también

[MADR-VAL_H31_38]

11) yo con toda esta gente bueno yo vine aquí y como la digo habían / toda la gente era mayor

[MADR-VAL_M31_41]

Por último, se ha documentado también un caso en el que la concordancia afecta no solo al número sino también a la persona. Se trata de la primera persona del plural del imperfecto, habíamos ${ }^{6}$.

6 Para algunos autores (Hernández Díaz 2006, Claes 2014a) la pluralización en primera de plural debe considerarse como un caso diferente de concordancia de haber existencial. Según este planteamiento, se trata de un tipo diferente de concordancia, ya que en ella se ve involucrado no solo el número sino también la persona y, mediante ella, el hablante se incluye en la enunciación y participa en la clase que se describe o caracteriza en la oración. Hernández Díaz considera que esta innovación de haber es en realidad una "refuncionalización de una forma ya existente" (2006:1153). No obstante, este autor había incluido los casos de habíamos o habemos en la tercera etapa de la difusión del fenómeno. 
12) nos man mandaron bajar a todos / que había un / un sótano y allí nos encerraron en la / en la caja fuerte a las siete u ocho personas que habíamos

[MADR-SAL_H33_15]

En resumen, la concordancia en plural, por tanto, solo se encuentra en dos tiempos verbales, el pretérito imperfecto, con diez ejemplos, y el pretérito perfecto compuesto, con dos. Y respecto a la estructura en la que se encuentra el verbo haber, solo en dos casos aparece como parte de una perífrasis, lo que está en consonancia también con el número de ocasiones en que se emplea perífrasis para la impersonalización. Aparece, además, un caso de concordancia en primera persona de plural (ejemplo 12).

Si se ponen en relación estos resultados con las etapas planteadas por Hernández Díaz (2006) para la difusión de la innovación, se puede decir que los datos de Madrid no se ajustan a la hipótesis, ya que no hay correspondencia entre la escasísima difusión social del fenómeno y el estadio evolutivo en que se encontraría. Los datos indican que la pluralización habría alcanzado las primeras fases, que son las que implican una menor alteración sobre la forma verbal, ya sea porque el cambio afecta a un verbo diferente (caso de las perífrasis) o porque la pluralización supone una alteración mínima, la adición de un solo fonema. Pero la presencia de habíamos significa que se ha llegado ya a la fase en la que las alteraciones en la forma verbal son más notables.

Los datos obtenidos, por otro lado, se ajustan solo en parte a la hipótesis de Claes (2014a) de la "disuasión estadística" como principio restrictor de la selección de la concordancia en plural. Como prevé la hipótesis, no se producen casos de pluralización de hay ni de hubo, por ser formas verbales estrechamente asociadas al valor presentacional o existencial. Asimismo, el mayor número de casos de pluralización se da en la forma había, que aparece en diversas construcciones. No obstante, la hipótesis no explica que no aparezca ningún caso en formas como habría o hubiera que, como habia, aparecen en español en varias construcciones y no están asociadas al valor presentacional. Tampoco permite explicar por qué la pluralización se ha extendido también al pretérito perfecto compuesto.

Tabla 4. Haber existencial + FN plural. Variables lingüísticas

\begin{tabular}{lllllllll}
\hline Grupo & Factor & $\begin{array}{l}\text { No } \\
\text { concord. }\end{array}$ & Concord. $\%$ & Total & $\chi^{2}$ & $p$ \\
\hline Estructura & No perífrasis & 508 & 98,1 & 10 & 1,9 & 518 & 0,944 & 0,331 \\
verbal & Perífrasis & 48 & 96,0 & 2 & 4,0 & 50 & & \\
\hline
\end{tabular}




\begin{tabular}{|c|c|c|c|c|c|c|c|}
\hline \multirow{4}{*}{$\begin{array}{l}\text { Valor } \\
\text { semántico del } \\
\text { argumento }\end{array}$} & $\mathrm{N}$ concreto & 375 & $98,2 \quad 7$ & 1,8 & 382 & \multirow{4}{*}{\multicolumn{2}{|c|}{$4,691 \quad 0,196$}} \\
\hline & $\mathrm{N}$ no concreto & 104 & $98,1 \quad 2$ & 1,9 & 106 & & \\
\hline & $\begin{array}{l}\text { Tiempo } \\
\text { cronológico }\end{array}$ & 55 & $98,2 \quad 1$ & 1,8 & 56 & & \\
\hline & $\begin{array}{l}\text { Tiempo } \\
\text { atmosférico }\end{array}$ & 22 & $91,7 \quad 2$ & 8,3 & 24 & & \\
\hline \multirow{2}{*}{$\begin{array}{l}\text { Animacidad } \\
\text { del argumento }\end{array}$} & Humano & 96 & $97,0 \quad 3$ & 3,0 & 99 & \multirow[t]{2}{*}{0,488} & 0,485 \\
\hline & No humano & 460 & $98,1 \quad 9$ & 1,9 & 469 & & \\
\hline \multirow{2}{*}{$\begin{array}{l}\text { Definitud del } \\
\text { argumento }\end{array}$} & Definido & 51 & $94,4 \quad 3$ & 5,6 & 54 & \multirow[t]{2}{*}{3,420} & 0,064 \\
\hline & No definido & 505 & $98,2 \quad 9$ & 1,8 & 514 & & \\
\hline \multirow[t]{3}{*}{ Forma léxica } & Sustantivo & 480 & $98,2 \quad 9$ & 1,8 & 489 & \multirow[t]{3}{*}{4,455} & 0,108 \\
\hline & Pronombre & 47 & $94,0 \quad 3$ & 6,0 & 50 & & \\
\hline & Implícita & 29 & 100,00 & 0,0 & 29 & & \\
\hline \multirow{3}{*}{$\begin{array}{l}\text { Posición del } \\
\text { argumento }\end{array}$} & Antepuesto & 42 & $93,3 \quad 3$ & 6,7 & 45 & \multirow[t]{3}{*}{5,405} & 0,067 \\
\hline & Pospuesto & 481 & $98,2 \quad 9$ & 1,8 & 490 & & \\
\hline & Elidido & 33 & 100,00 & 0,0 & 33 & & \\
\hline \multirow{3}{*}{$\begin{array}{l}\text { Marca de } \\
\text { pluralidad en } \\
\text { el argumento }\end{array}$} & $\mathrm{N}$ escueto & 252 & $97,7 \quad 6$ & 2,3 & 258 & \multirow[t]{3}{*}{0,349} & 0,840 \\
\hline & $\begin{array}{l}\mathrm{N} \text { con } \\
\text { adjuntos }\end{array}$ & 291 & $98,0 \quad 6$ & 2,0 & 297 & & \\
\hline & $\mathrm{N}$ colectivo & 13 & 100,00 & 0,0 & 13 & & \\
\hline \multirow{8}{*}{$\begin{array}{l}\text { Tiempo y } \\
\text { modo verbal }\end{array}$} & Pres. indic. & 35 & 100,00 & 0,0 & 35 & \multirow[t]{8}{*}{4,233} & 0,753 \\
\hline & Pret. imp. Ind. & 325 & $97,0 \quad 10$ & 3,0 & 335 & & \\
\hline & $\begin{array}{l}\text { Pret. perf. } \\
\text { simple }\end{array}$ & 39 & 100,00 & 0,0 & 39 & & \\
\hline & $\begin{array}{l}\text { Pret. perf. } \\
\text { comp. ind. }\end{array}$ & 92 & $97,9 \quad 2$ & 2,1 & 94 & & \\
\hline & Futuro & 21 & 100,00 & 0,0 & 21 & & \\
\hline & Condicional & 3 & 100,00 & 0,0 & 3 & & \\
\hline & Subjuntivo & 40 & 100,00 & 0,0 & 40 & & \\
\hline & Infinitivo & 1 & 100,00 & 0,0 & 1 & & \\
\hline \multirow{2}{*}{$\begin{array}{l}\text { Intercalación } \\
\text { de elementos }\end{array}$} & Ausencia & 525 & $97,9 \quad 11$ & 2,1 & 536 & \multirow[t]{2}{*}{0,168} & 0,682 \\
\hline & Presencia & 31 & $96,9 \quad 1$ & 3,1 & 32 & & \\
\hline
\end{tabular}




\begin{tabular}{|c|c|c|c|c|c|}
\hline \multirow[t]{4}{*}{ Tipo de oración } & Principal & 258 & $99,2 \quad 2$ & $0,8 \quad 260$ & $7,974 \quad 0,047$ \\
\hline & Coordinada & 102 & $98,1 \quad 2$ & $1,9 \quad 104$ & \\
\hline & $\begin{array}{l}\text { Subord. no } \\
\text { adjetiva }\end{array}$ & 156 & $96,9 \quad 5$ & $3,1 \quad 161$ & \\
\hline & $\begin{array}{l}\text { Subord. } \\
\text { adjetiva }\end{array}$ & 40 & $93,0 \quad 3$ & $7,0 \quad 43$ & \\
\hline \multirow[t]{2}{*}{ Polaridad } & Afirmativa & 484 & $97,6 \quad 12$ & $2,4 \quad 496$ & $1,780 \quad 0,182$ \\
\hline & Negativa & 72 & 100,00 & $0,0 \quad 72$ & \\
\hline Total & & 556 & $97,9 \quad 12$ & 2,1568 & \\
\hline
\end{tabular}

Respecto a las características semánticas del argumento con que se combina el verbo, tres remiten a un referente humano y nueve a entidades no animadas; entre estas últimas hay dos referidas a tiempo atmosférico (nevadas) y una a tiempo cronológico (épocas); el resto son siete sustantivos concretos y dos no concretos. Nueve de los argumentos se actualizan con sustantivos y tres con pronombres, los tres relativos que encabezan la oración correspondiente. Las entidades que seleccionaron los hablantes como sujetos de las respectivas oraciones son bulevares, compañeros, cuevas, épocas, gente, hoteles, nevadas, nosotros, oposiciones, problemas, puentecitos y tormentas. Si clasificamos este grupo siguiendo la propuesta de Claes (2014a) de establecer la agentividad no según el rasgo \pm humano, sino según la capacidad de la entidad para ser "cabeza de la cadena de acciones", los elementos más agentivos son, además del pronombre nosotros, los sustantivos animados compañeros, gente, y los sustantivos inanimados tormentas y problemas; en la "cola de la cadena de acciones" se sitúan oposiciones, nevadas, épocas, bulevares, cuevas, hoteles y puentecitos. Es decir, de los doce casos de pluralización de haber hay cinco que se ajustan al prototipo de sujeto, mientras que siete se alejan de los rasgos con los que prototípicamente se asocia el sujeto. A pesar del reducido número de casos, los datos de Madrid no se ajustan al modelo esperado, ya que los hablantes seleccionaron el sujeto de estas oraciones en función de criterios diferentes del grado de agentividad de las entidades.

El sintagma que forma el complemento en tres ocasiones está definido mediante algún tipo de determinante y en nueve aparece como no definido y ese mismo reparto se produce respecto a la posición que ocupa: pospuesto nueve veces y antepuesto tres. En cambio, hay equilibrio en cuanto a las marcas de pluralidad que acompañan al sustantivo, que se reparten: seis para los casos sin modificadores y otras tantas en los que el sustantivo está modificado. El complemento se sitúa en todas las ocasiones adosado directamente al verbo, excepto en una (ej. 6). 
Respecto a las características de la oración en la que se sitúan los casos de concordancia, todos ellos se encuentran en oraciones afirmativas, y se distribuyen entre las oraciones principales, con dos ejemplos, las coordinadas, con otros dos, las subordinadas adjetivas, con tres casos, y el resto de subordinadas, con cinco casos. En este punto solo llama la atención el porcentaje más elevado que suponen los casos de concordancia en las subordinadas adjetivas, donde se alcanza el 7\% frente a las demás variantes, que no superan el $3 \%$. De hecho, las pruebas de estadística inferencial señalan que este factor lingüístico es significativo en la comunidad de Madrid $\left(\chi^{2}=\right.$ $7,974(3 \mathrm{gl}), \mathrm{p}=0,047) \mathrm{y}$, aunque la correlación entre las variables es baja, también ha resultado estadísticamente significativa $(\mathrm{V}$ de $\mathrm{Cramer}=0,118$, $\mathrm{p}=0,047)$.

\subsection{LAS VARIABLES ESTILÍSTICAS}

Las características estilísticas de estos ejemplos muestran que todos los casos aparecen en el discurso no especializado, pero hay que tener en cuenta que este tipo de discurso es el más frecuente en las encuestas sociolingüísticas; de hecho es prácticamente el único que se da en ellas. Cinco casos se encuentran en textos de carácter expositivo-explicativo, cuatro en narraciones, dos en descripciones y uno en textos argumentativos. Y en cuanto a la relación que se establece entre los interlocutores, cuando el estatus del encuestado es menor que el del entrevistador es más frecuente la pluralización y lo mismo sucede cuando la edad del encuestado es mayor que la del encuestador.

Tabla 5. Haber existencial + FN plural. Variables estilísticas

\begin{tabular}{llccccccc}
\hline Grupo & Factor & $\begin{array}{c}\text { No } \\
\text { concord. }\end{array}$ & $\%$ & Concord. & $\%$ & Total & $\square^{2}$ & $\mathrm{p}$ \\
\hline Especialización & Cotidiano & 544 & 97,8 & 12 & 2,2 & 556 & 0,265 & 0,607 \\
del discurso & Especializado & 12 & 100,0 & 0 & 0,0 & 12 & & \\
\hline Tipo de discurso & Expositivo- & 337 & 98,5 & 5 & 1,5 & 342 & 4,272 & 0,234 \\
& explicativo & & & & & & & \\
& Narrativo & 79 & 95,2 & 4 & 4,8 & 83 & & \\
& Argumentativo & 79 & 98,8 & 1 & 1,3 & 80 & & \\
& Descriptivo & 61 & 96,8 & 2 & 3,2 & 63 & & \\
\hline Tenor y estatus & Solidaridad & 92 & 97,9 & 2 & 2,1 & 94 & 0,000 & 0,991 \\
& Inferioridad & 464 & 97,9 & 10 & 2,1 & 474 & & \\
\hline
\end{tabular}




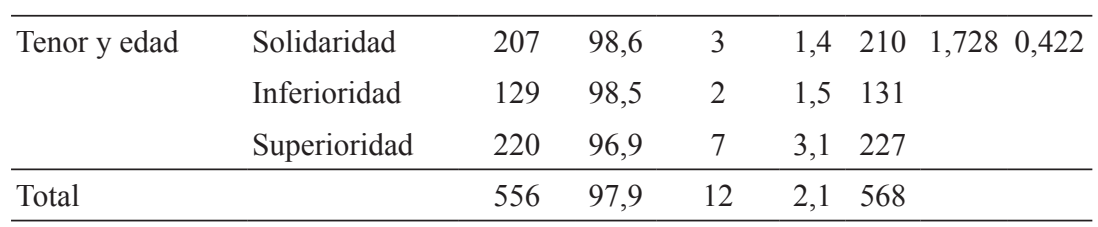

\subsection{LAS VARIABLES SOCIOLÓGICAS}

Por último, en cuanto a los factores sociales, por sexo destaca el predominio de los hombres, a quienes corresponden nueve ejemplos, sobre las mujeres. Con las reservas señaladas por la escasez de ocurrencias del fenómeno, el hecho apunta de nuevo en la línea de las investigaciones sociolingüísticas que señalan la mayor inclinación del grupo femenino a seguir los usos socialmente prestigiosos. En cuanto a la edad, hay diferencias entre grupos: los mayores de 55 años son quienes más casos de pluralización han realizado y con 7 casos se sitúan más del doble por encima del resto de grupos. Por el contrario, el nivel de estudios supone una igualdad completa entre los tres grupos considerados, cada uno de los cuales produjo cuatro casos. Y en cuanto a la clase social a la que pertenecen los encuestados, si atendemos solo al número de casos, se observa que el número va aumentando a medida que desciende la escala social, pero si atendemos a los porcentajes relativos de cada grupo, las dos clases situadas en los extremos obtienen los valores más elevados. Por último, los casos están prácticamente distribuidos en los dos distritos de residencia del hablante que se han tenido en cuenta.

Las características sociológicas de los individuos, en definitiva, no permiten ir más allá de los indicios que los datos pueden mostrar. Las pruebas de estadística inferencial han descartado que puedan extraerse conclusiones significativas a partir de los datos.

Tabla 6. Haber existencial + FN plural. Variables sociológicas

\begin{tabular}{llccccccc}
\hline Grupo & Factor & $\begin{array}{c}\text { No } \\
\text { concord. }\end{array}$ & $\%$ & Concord. & $\%$ & Total & $\square^{2}$ & $p$ \\
\hline Sexo & Hombre & 285 & 96,9 & 9 & 3,1 & 294 & 2,652 & 0,103 \\
& Mujer & 271 & 98,9 & 3 & 1,1 & 274 & & \\
\hline Edad & $20-34$ años & 125 & 98,4 & 2 & 1,6 & 127 & 1,492 & 0,474 \\
& $35-54$ años & 204 & 98,6 & 3 & 1,4 & 207 & & \\
& 55 años o más & 227 & 97,0 & 7 & 3,0 & 234 & & \\
\hline
\end{tabular}




\begin{tabular}{llccccccc}
\hline Nivel de & Primarios & 176 & 97,8 & 4 & 2,2 & 180 & 0,046 & 0,977 \\
estudios & Medios & 178 & 97,8 & 4 & 2,2 & 182 & & \\
& Universitarios & 202 & 98,1 & 4 & 1,9 & 206 & & \\
\hline Modo de vida & Familia & 107 & 99,1 & 1 & 0,9 & 108 & 1,956 & 0,376 \\
& Ocio & 414 & 97,4 & 11 & 2,6 & 425 & & \\
& Trabajo & 35 & 100,0 & 0 & 0,0 & 35 & & \\
\hline Clase social & Baja & 98 & 96,1 & 4 & 3,9 & 102 & 2,993 & 0,393 \\
& Media-baja & 157 & 98,1 & 3 & 1,9 & 160 & & \\
& Media & 242 & 98,8 & 3 & 1,2 & 245 & & \\
& Media-alta & 59 & 96,7 & 2 & 3,3 & 61 & & \\
\hline Distrito de & Salamanca & 233 & 97,9 & 5 & 2,1 & 238 & 0,000 & 0,987 \\
residencia & & & & & & & & \\
\hline & Vallecas & 323 & 97,9 & 7 & 2,1 & 330 & & \\
\hline Total & & 556 & 97,9 & 12 & 2,1 & 568 & & \\
\hline
\end{tabular}

\subsection{EL PAPEL DEL INDIVIDUO EN LA VARIACIÓN}

Por último, hemos querido analizar las características individuales de los hablantes que han pluralizado el verbo. Interesan aspectos como cuántos son, qué origen tienen, su vinculación con las zonas hispanohablantes en las que el proceso de pluralización está más avanzado, si el uso concordado puede deberse a su participación en el mercado lingüístico o el grado de variación intraindividual que muestran.

\begin{tabular}{llccccccc}
\hline Grupo & Factor & $\begin{array}{c}\text { No } \\
\text { concord. }\end{array}$ & $\%$ & Concord. & $\%$ & Total & $\square^{2}$ & $\mathrm{p}$ \\
\hline Origen & Madrileño & 414 & 97,4 & 11 & 2,6 & 425 & 2,039 & 0,361 \\
& Vallecano & 52 & 100,0 & 0 & 0,0 & 52 & & \\
& Inmigrante & 90 & 98,9 & 1 & 1,1 & 91 & & \\
\hline Ascendencia & De zona & 506 & 97,7 & 12 & 2,3 & 518 & 1,183 & 0,277 \\
& no concord. & & & & & & & \\
& De zona & 50 & 100,0 & 0 & 0,0 & 50 & & \\
& concordante & & & & & & & \\
\hline Total & & 556 & 97,9 & 12 & 2,1 & 568 & & \\
\hline
\end{tabular}


Prácticamente todos los casos de pluralización corresponden a sujetos nacidos en Madrid: solo un ejemplo concordado en plural corresponde a una persona nacida fuera de la ciudad y ninguno de los vallecanos recurre a esta construcción sintáctica. Los 12 casos de concordancia en plural que se han venido analizando aparecen en las encuestas $8,15,19$ y 35 , que corresponden a residentes en el Distrito de Salamanca, y las encuestas 12, 26, 32, 38 y 41, de sujetos de los distritos de Vallecas o Puente de Vallecas. Por tanto, el grupo de hablantes que concentran los casos de pluralización está constituido por nueve hablantes, lo que supone un porcentaje de $8,3 \%$ sobre el total de las 108 personas encuestadas en los dos distritos madrileños.

Estos sujetos constituyen, por tanto, la avanzadilla de la variación y lideran el cambio (Martín Butragueño 2006). El grupo es heterogéneo: está constituido por tres mujeres y seis hombres; hay dos de la primera generación, tres de la segunda y cuatro de la tercera; por nivel de estudios, dos tienen estudios primarios, tres han cursado estudios secundarios y otros tres tienen estudios universitarios. Todos ellos, menos uno, se encuadran en el tipo de vida "ocio" y también todos ellos han nacido en Madrid, salvo una mujer, natural de la provincia de Guadalajara. En cuanto a las profesiones que desempeñan, hay tres vinculadas con el mercado lingüístico (un comercial, una estudiante y teleoperadora y un auxiliar de farmacia) y cinco no vinculadas con el mercado lingüístico (un maestro de laboratorio, un operario de una compañía aérea, un jubilado que fue empleado municipal, una modista y una ama de casa) y no se dispone de datos de uno de ellos.

En definitiva, el perfil que presentan estos datos muestra que el hablante que introduce la pluralización en Madrid es el siguiente:

Varón de la tercera generación y estudios secundarios, nacido en Madrid y sin especial vinculación con regiones concordantes, que trabaja por cuenta ajena en una profesión para la que no requiere especialmente el dominio lingüístico.

No obstante, ninguno de estos hablantes innovadores selecciona la variante pluralizada en todas las ocasiones (Gráfico 1). Al contrario, la pluralización del verbo es siempre una solución minoritaria: de hecho, en todos ellos solo se encuentra un caso, salvo el hombre de la encuesta 15, que presenta dos, y el de la encuesta 104, que concordó en plural en tres ocasiones. 


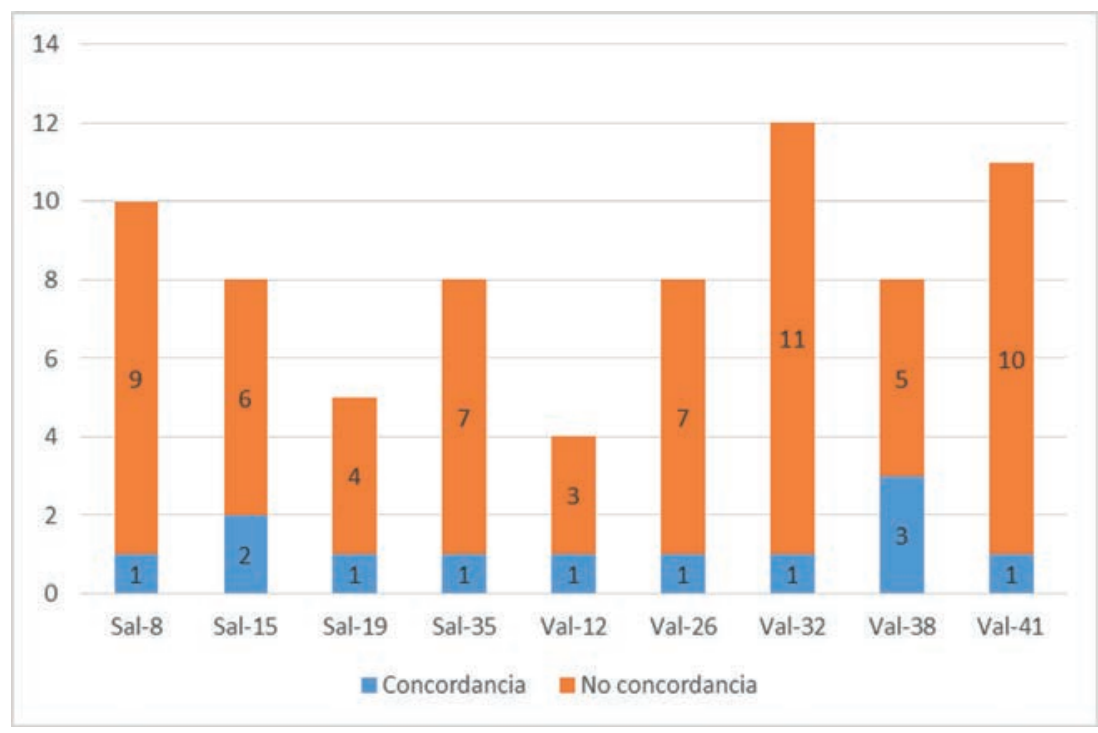

Gráfico 1. Relación de haber existencial pluralizado y no pluralizado por individuo

En resumen, tanto el hecho de que los hablantes innovadores sean naturales de Madrid, sin vinculación con sujetos originarios de áreas en las que se produce la pluralización, hace suponer que el cambio se ha producido no mediante los mecanismos habituales del cambio cara a cara, sino mediante otras formas de contacto con otras formas de habla. En este sentido cabría pensar que han sido los medios de comunicación de masas, como se ha sugerido (RAE y ASALE 2009) el instrumento a través del que se difunde esta innovación lingüística.

A la vista de los datos todavía cabe hacerse una última pregunta: ¿tiene algún efecto el priming estructural en el comportamiento lingüístico de los madrieños que han adoptado la construcción pluralizada? El hecho de que los hablantes por regla general solo hayan producido un caso de construcción concordada en plural es un argumento en contra de que en la comunidad de habla madrileña influya el efecto priming, y en este sentido hay que interpretar también la autocorrección que se observa en el ejemplo 8 (vid § 4.1). Por si ello fuera poco, el número de casos en total es un último argumento para suponer que no tiene influencia, al menos en la fase en la que el fenómeno se encuentra. 


\section{CONCLUSIONES}

Las conclusiones que podemos extraer de este trabajo son necesariamente provisionales, dadas las reservas que impone el escaso número de ejemplos encontrados a cualquier generalización. No obstante, precisamente por su carácter iniciático, se pueden apuntar algunas ideas sobre el modo en que se está produciendo la pluralización de haber en Madrid.

En primer lugar, se constata que el fenómeno está presente en Madrid, aunque de forma embrionaria. Como hemos visto, la pluralización supone tan solo el $2,1 \%$ del total de casos de haber existencial, como corresponde a los momentos iniciales de cualquier cambio lingüístico. Los valores porcentuales ascienden algo más si se toma en cuenta el número de madrileños que recurren a estas construcciones: 9 de los 108 encuestados han acogido ya esta construcción sintáctica, lo que eleva el porcentaje hasta el 8,3\% de la muestra.

Desde el punto de vista evolutivo, se puede hablar de que la innovación sintáctica va avanzando, aunque de manera lenta, no solo por el número de casos y contextos en los que se encuentra hoy, sino especialmente porque afecta a todos los estratos sociales, incluido el culto, en el que nunca o casi nunca aparecía treinta años atrás (De Mello 1991, Quilis 1983). Tomados idénticos sectores poblacionales, es decir, seleccionando solo los hablantes de instrucción universitaria de las encuestas del PRESEEA y el total de casos de haber existencial, incluidos los de hay como ocurre en los datos de la norma culta, se constata que la innovación avanza. En las encuestas de finales de los 60 y principios de los 70, encontramos dos casos de 2385, es decir, el 0,08\%, que corresponden también a solo dos hablantes de los 179 encuestados, es decir, el 1,11\%. En los datos actuales, han ascendido tanto los datos relativos al total de casos (4 de 1285, que representa el 0,31\%) como al número de hablantes ( 3 de los 36 universitarios, es decir, el 8,3\%).

En la lentitud del avance de la innovación tiene mucho que ver el freno de las presiones normativas que repudian este uso, como refleja la mayor resistencia a la innovación por parte de los grupos que tradicionalmente se oponen a los cambios menos prestigiosos, las mujeres y los grupos más instruidos.

Los datos de Madrid no se ajustan a la hipótesis de las fases del cambio que supone que la innovación va ligada al grado de afectación de la forma fónica de haber. Los ejemplos aportados en este estudio muestran que, aunque el cambio se ha introducido en aquellas formas que suponen una modificación menor, ya sea porque el plural aparece en una forma 
perifrástica, ya porque el cambio supone la adición de un solo sonido (habian, han habido), también se ha encontrado un caso de pluralización de la primera persona de plural (habíamos), que supone una alteración mayor y que no se esperaría hasta un momento de mayor difusión social y contextual de la construcción innovadora. Así pues, aunque en el plano estrictamente lingüístico no puede descartarse el valor explicativo de la gradualidad del cambio, la hipótesis no parece cumplirse cuando se trata de la adopción por parte de hablantes de una comunidad de habla específica.

Del mismo modo, los resultados madrileños tampoco favorecen las hipótesis de las restricciones cognitivas que teóricamente actúan sobre la aparición de la construcción: no hay evidencias de que tenga algún tipo de efecto el priming estructural en la presencia de los escasos ejemplos hallados, ni tampoco parece que afecte la marcadez de la construcción a la luz de las características semánticas de las FN que funcionan como sujeto cuando el hablante selecciona la construcción pluralizada. Respecto a la restricción debida a la disuasión estadística, solo se observa parcialmente, ya que las formas verbales afectadas no se restringen a las que forman esquemas cognitivos más estables para la expresión de la existencia.

En el proceso de difusión, la innovación no va asociada abiertamente a ningún factor estilístico ni social y, entre los lingüísticos, solo el tipo de oración parece incidir en la presencia de las construcciones pluralizadas de haber. La concordancia en plural se produce algo más en oraciones de relativo siempre que estas estén introducidas por que como sujeto, lo que hace preguntarse si tendrá algo que ver el hecho de que este pronombre esté incapacitado para portar marcas de plural. Por otra parte, la variación se produce en individuos que no tienen especial vinculación con las áreas que tradicionalmente han sido señaladas como zonas concordantes ni que necesiten especial dominio de los recursos lingüísticos para su actividad social. Ello hace pensar que la difusión de la innovación ha comenzado a través de mecanismos de difusión diferentes del contacto tradicional cara a cara, quizá como consecuencia de los modelos presentados a través de los medios de comunicación.

Una última reflexión es que los datos de Madrid apuntan a que el cambio, de producirse, no se realizará de manera gradual, sino siguiendo un patrón abrupto, en el que se pueden ver afectadas simultáneamente todas las estructuras gramaticales en las que participe el verbo haber, con la salvedad de aquellas formas que suponen el grado más extremo. La suerte que corra la construcción pluralizada en Madrid dependerá del modo en que se perciba esta innovación, hoy por hoy muy estigmatizada, y de las actitudes que los madrileños adopten ante ella. Es esta una cuestión que ha de ser abordada como tarea para el futuro inmediato. 


\section{REFERENCIAS BIBLIOGRÁFICAS}

Almeida, Manuel y Carmen Díaz Alayón. 1989. El español de Canarias. Santa Cruz de Tenerife: Edic. de los autores.

Bentivoglio, Paola y Mercedes Sedano. 1989. Haber: ¿un verbo impersonal? Estudios sobre el español de América y lingüistica afro-americana. Ponencias presentadas en el 45 congreso internacional de americanistas, pp. 59-81. Bogotá: Instituto Caro y Cuervo.

Blas Arroyo, José Luis. 2005. Sociolingüistica del español. Desarrollos y perspectivas en el estudio de la lengua española en contexto social. Madrid: Cátedra.

2016. Entre la estabilidad y la hipercorrección en un antiguo 'cambio desde abajo': Haber existencial en las comunidades de habla castellonenses. Disponible en https:// www.academia.edu/24370590/Entre_la_estabilidad_y_la_hipercorrecci\%C3\%B3n_en un_antiguo_cambio_desde_abajo_Haber_existencial_en_las_comunidades_de_habla castellonenses [Consulta 29/05/2016]

Catalán, Diego. 1964. El español en Canarias. Presente y futuro de la lengua española. vol. I, pp. 239-280. Madrid: Ediciones Cultura Hispánica,.

Cestero, A. María, Isabel Molina y Florentino Paredes. 2015. Patrones sociolingüísticos de Madrid. Bern: Peter Lang.

Claes, Jeroen. 2014a. La pluralización de haber presentacional en el español de La Habana: interacción entre las restricciones cognitivas. Revista Internacional de Lingüística Iberoamericana 23: 165-187.

2014b. Sociolingüística comparada y gramática de construcciones: Un acercamiento a la pluralización de haber presentacional en las capitales antillanas. Revista Española de Lingüística Aplicada 27/2: 338-364.

D’Aquino, Giovanna. 2004. Haber impersonal en el habla de Caracas: análisis sociolingüístico. Boletín de Lingüística 21: 3-26.

De Mello, George. 1991. Pluralización del verbo haber impersonal en español hablado culto de once ciudades, Thesaurus 46: 445-471.

Fernández Soriano, Olga y Susana Táboas Baylín. 1999. Construcciones impersonales no reflejas. En I. Bosque y V. Demonte (dirs.). Gramática Descriptiva de la Lengua Española, pp. 1723-1778. Madrid: Espasa.

Flórez, LuIs. 1946. Reseña a Charles E. Kany. American Spanish Syntax. Thesaurus 2: 372-385.

Fontanella de Weinberg, María Beatriz. 1992. Variación sincrónica y diacrónica de las construcciones con haber en el español americano. Boletín de Filología de la Universidad de Chile 33: 35-46.

Goldberg, Adele E. 2006. Constructions at work: The nature of generalization in language. Oxford: Oxford University Press.

Gómez Molina, José Ramón. 2013. Pluralización de haber impersonal en el español de Valencia (España). En José Ramón R. Gómez Molina (coord.). El español de Valencia. Estudio sociolingüístico, pp. 109-143. Bern: Peter Lang.

GonzÁlez Calvo, José Manuel. 2002. Sintaxis y semántica: Haber impersonal en español. En M. Velarde, Pulchre, bene, recte: Homenaje al Prof. Fernando González-Ollé, pp. 639-656. Pamplona: Universidad de Navarra.

Hernández Díaz, Axel. 2006. Posesión y existencia. La competencia de haber, tener en la posesión y haber existencial. En Concepción Company Company (ed.). Sintaxis histórica de la lengua española. Primera parte: la frase verbal, vol. 2, pp. 1053-1160. México: Universidad Nacional Autónoma de México / Fondo de Cultura Económica. 
Kany, Charles E. 1971. Sintaxis hispanoamericana. Madrid: Gredos.

Langacker, Ronald W. 1991. Foundations of cognitive grammar. Volume 2: Descriptive application. Stanford: Stanford University Press.

Lastra, Yolanda y Pedro Martín Butragueño. 2015. La concordancia de haber existencial en la ciudad de México. CILIR2015. XV Coloquio Internacional de Lingüística Iberorromance. Rouen, 3-5 de junio. Disponible en https://www.academia.edu/12688928/ La_concordancia_de haber_existencial_en_la_ciudad_de_M\%C3\%A9xico_Y._Lastra_ and_P._Mart\%C3\%ADn_draft_1 [Consulta 27/05/2016]

Llorente, Antonio. 1980. Consideraciones sobre el español actual. Anuario de Letras 18: 5-61.

Lope Blanch, Juan Manuel. 1953. Observaciones sobre la sintaxis del español hablado en México. México: UNAM.

Lorenzo EMiLIo. 1971. El español de hoy: lengua en ebullición. Madrid: Gredos.

Luque Moreno, Jesús. 1978. En torno al sintagma 'haber impersonal + sustantivo' y sus orígenes latinos. Revista Española de Lingüistica 8/1: 125-146.

Martín Butragueño, Pedro. 2006. Líderes lingüísticos en la ciudad de México. En P. Martín Butragueño (ed.). Lideres lingüísticos, Estudios de variación y cambio, pp.185-208. México: El Colegio de México.

Martínez, José Antonio. 1999. La concordancia. En I. Bosque y V. Demonte (dirs.). Gramática descriptiva de la Lengua Española, pp. 2695-2786. Madrid: Espasa.

Montes Giraldo, José J. 1982. Sobre el sintagma haber + sustantivo. Thesaurus. Boletín del Instituto Caro y Cuervo 37: 383-385.

Moreno Fernández, Francisco. 1996. Metodología del 'Proyecto para el estudio sociolingüístico del Español de España y de América' (PRESEEA). Lingüística 8: 257-287.

PRESEEA. 2013. Proyecto para el estudio sociolingüístico del español de España y América, "Codificación de la variable «usos del verbo haber» (singular/plural)", en $<$ http://preseea. linguas.net/Metodolog\%C3\%ADa.aspx>. [Consulta 20/04/2016]

Quilis, ANToNio. 1983. La concordancia gramatical en la lengua española hablada en Madrid. Madrid: C.S.I.C.

Real Academia Española y Asociación de Academias de la Lengua Española. 2009. Nueva gramática de la lengua española. Madrid: Espasa-Calpe.

Regueiro Rodríguez, M. ${ }^{a}$ Luisa. 2008. El español en la prensa, aspectos morfosintácticos. En Antonio Arroyo (coord.). La lengua española en los medios de comunicación y en las nuevas tecnologías, pp. 67-132. Madrid: Laberinto.

Samper Padilla, José A. y Clara E. Hernández. 2012. En torno a los usos personales de haber en el español de Las Palmas de Gran Canaria. En Tomás Jiménez Juliá et alii (eds.). Cum corde et in nova grammatica. Estudios ofrecidos a Guillermo Rojo, pp. 743-754. Santiago de Compostela: Universidad de Santiago de Compostela.

Seco Reymundo, Manuel. 1986. Diccionario de dudas y dificultades de la lengua española. Madrid: Espasa Calpe. 